\title{
Sex Difference and Senile Change in Hypothalamic Neuronal Response to Electrical Stimulation of the Limbic System in the Rat
}

\author{
TATSuo AKEMA and Masazumi KAWAKAMI \\ Department of Physiology Yokohama City University School of Medicine \\ 2-33 Urafune-cho, Minami-ku, Yokohama 232
}

\begin{abstract}
To get an electrophysiological basis for understanding the sexual and age-related changes in the brain mechanisms controlling gonadotropin secretion, we studied single unit responses to electrical stimulation in the preoptico-arcuate, amygdalo-preoptic and hippocampo-preoptic neural connections in the rat. Orthodromic unit responses to single pulse electrical stimulation were recorded in young mature and aged, male and female rats by post-stimulus histogram analysis.

Electrical stimulation of the medial preoptic area elicited responses in one-half of the hypothalamic arcuate neurons tested in female rats while the stimulation induced responses in less than one-third of the arcuate neurons in the male. The percentage of arcuate neurons responding to preoptic stimulation did not differ between young and aged animals. Stimulation of the medial amygdala evoked responses in a larger number of preoptic neurons in young mature rats than in aged animals in both sexes. There was no sexual difference in the number of responding neurons to amygdaloid stimulation. Percentages of preoptic neurons responding to the hippocampal stimulation were greater in young females showing regular estrous cycles than in males or aged females which showed constant estrous vaginal smears. In aged females whose vaginal cycles were induced by daily treatment with progesterone, more preoptic neurons responded to stimulation of the amygdala and the hippocampus than in aged constant estrous rats. The young cycling and aged progesteronerecycling rats had similar preoptic neuronal responses to the limbic stimulation. Stimulus thresholds for inducing these arcuate and preoptic unit responses were almost equivalent among different groups of animals. This suggests that the alteration in unit responses observed was not due to a change in neuronal excitability in the sites of stimulation in different animal groups. These results indicate that there are sex differences in the synaptic functions which transmit preoptic signals to the arcuate neurons and hippocampal impulses to the preoptic neurons. It is also suggested that the amygdalo-preoptic and hippocampopreoptic neural pathways may be functionally modified in aged rats because of the absence of estrous cycles.
\end{abstract}

The reproductive functions in the male, female and aged rats are controlled by the central nervous system in apparently different ways. Cyclic gonadotropin release and ovulation, which are characteristic of mature females, do not occur in male animals orchidectomized and transplanted with ovaries. Aged females

Received July 29, 1982. also lose these cyclic functions. The loss of estrous cycles in aged rats is due to a functional alteration in the brain since the transplantation of ovaries or pituitaries from aged non-cycling rats to young mature cycling rats does not prevent the cycling activity in the young animals (Peng and Huang, 1972). Electrical stimulation of the preoptic area causes a rise in serum gonadotropin concentrations 
and ovulation in young female rats (Everett and Radford, 1961; Kawakami, et al., 1973b), aged constant-estrous rats (Clemens, et al., 1969) and in male animals (Quinn, 1966; Kawakami, et al., 1973a). However, the aged rat or the male with an ovarian transplant does not ovulate spontaneously, suggesting the possibility that afferent inputs to the preoptic area may be different in these animals from young mature females. Raisman and Field (1973) revealed a sexual difference in the morphological pattern of non-amygdaloid axon terminals on preoptic neurons. Dyer et al. (1976) reported a sexual dimorphism in the preoptic unit response to stimulation of the amygdala in the rat. To get more information about the sexual difference in the afferent influences on the preoptic and hypothalamic electrical activities, we examined the unit responses to electrical stimulation in the preoptico-arcuate, amygdalo-preoptic and hippocampo-preoptic systems in the rat. Unit responses were also recorded from aged females showing constant estrous vaginal smears with or without daily progesterone treatment which induced estrous cycles in constant estrous rats. Sexual differences were found in the neural functions transmitting impulses from the preoptic area to the arcuate neurons and from the hippocampus to the preoptic neurons. It was also found that the impulse-transmitting activity of the amygdalo-preoptic and the hippocampopreoptic pathways is modified in aged rats and accompanied by the loss of estrous cycles.

\section{Materials and Methods}

A hundred and eight male and female, young mature (3- to 6-month old) and aged (15- to 24-month old) rats of Wistar strain were used. They were housed under artificial light control (lights on 05.00-19.00 h) and fed laboratory chows ad libitum. Daily vaginal smears were taken from female animals and young females whose smears showed regular 4-day estrous cycles were used on the day of proestous or diestrous I. Aged females were used when they showed at least 21 days of constant vaginal estrous. Fourteen of the constant estrous females were injected with proges-
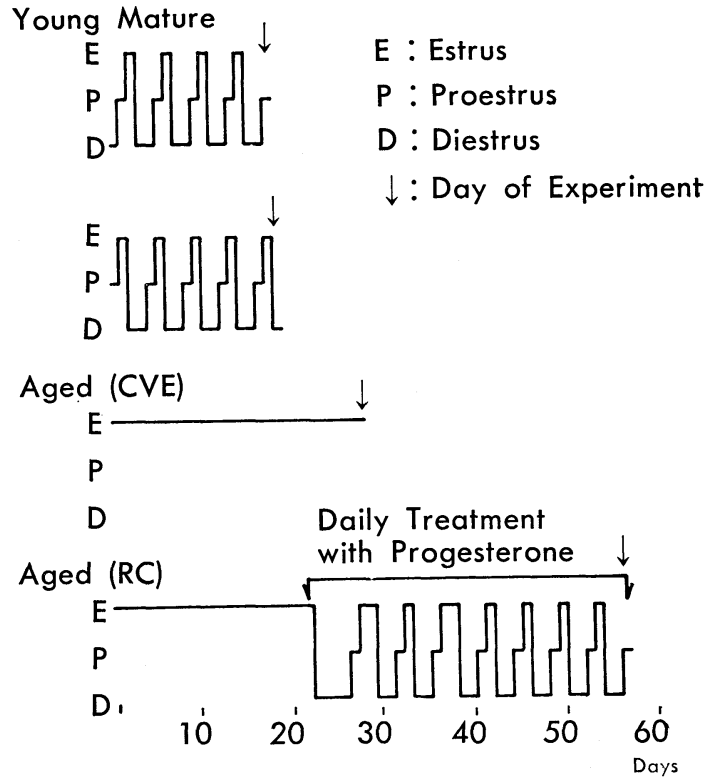

Fig. 1. Examples of daily vaginal smears from female rats. Young mature females were used on the day of proestrous or diestrous I. Old rats were used when their vaginal smears showed at least 21 days of persistent estrous, with or without progesterone daily injection which induced vaginal cycles in 11 of 14 persistent estrous rats. CVE: constant vaginal estrous. RC: recycling.

terone $(1 \mathrm{mg}$, s.c., daily) and in 11 of them vaginal cycles were induced after progesterone; the recycling old rats were used on the day of proestrous. Fig. 1 shows examples of the daily vaginal smears from female rats used in this study.

Rats were anesthetized with urethane $(1.25 \mathrm{~g} / \mathrm{kg}$ b.w., injected i.p.), and fixed in a stereotaxic apparatus. A stimulating bipolar concentric electrode, made from a stainless steel tube with a $0.4 \mathrm{~mm}$ outer diameter and a stainless steel wire $0.15 \mathrm{~mm}$ in diameter and insulated with epoxylite except the tip, was oriented to the medial preoptic area, medial amygdala or dorsal hippocampus according to the atlas of Albe-Fessard et al. (1966). Negative square wave pulses of constant intensity (0.1$2.0 \mathrm{~mA})$, duration $(0.2 \mathrm{msec})$ and frequency $(2 / 3 \mathrm{~Hz})$ were applied through the electrode.

Extracellular potentials were recorded via a glass micropipette made from a Pyrex tube with a $3 \mathrm{~mm}$ outer diameter and filled with $0.5 \mathrm{M}$ sodium acetate solution. Pontamine sky blue 6B was added to make a $2 \%$ solution of dye for marking the position of the tip of the micropipette. The micropipette was inserted into the hypothalamic arcuate nucleus or the medial preoptic area, and extracellular unit potentials were taken 


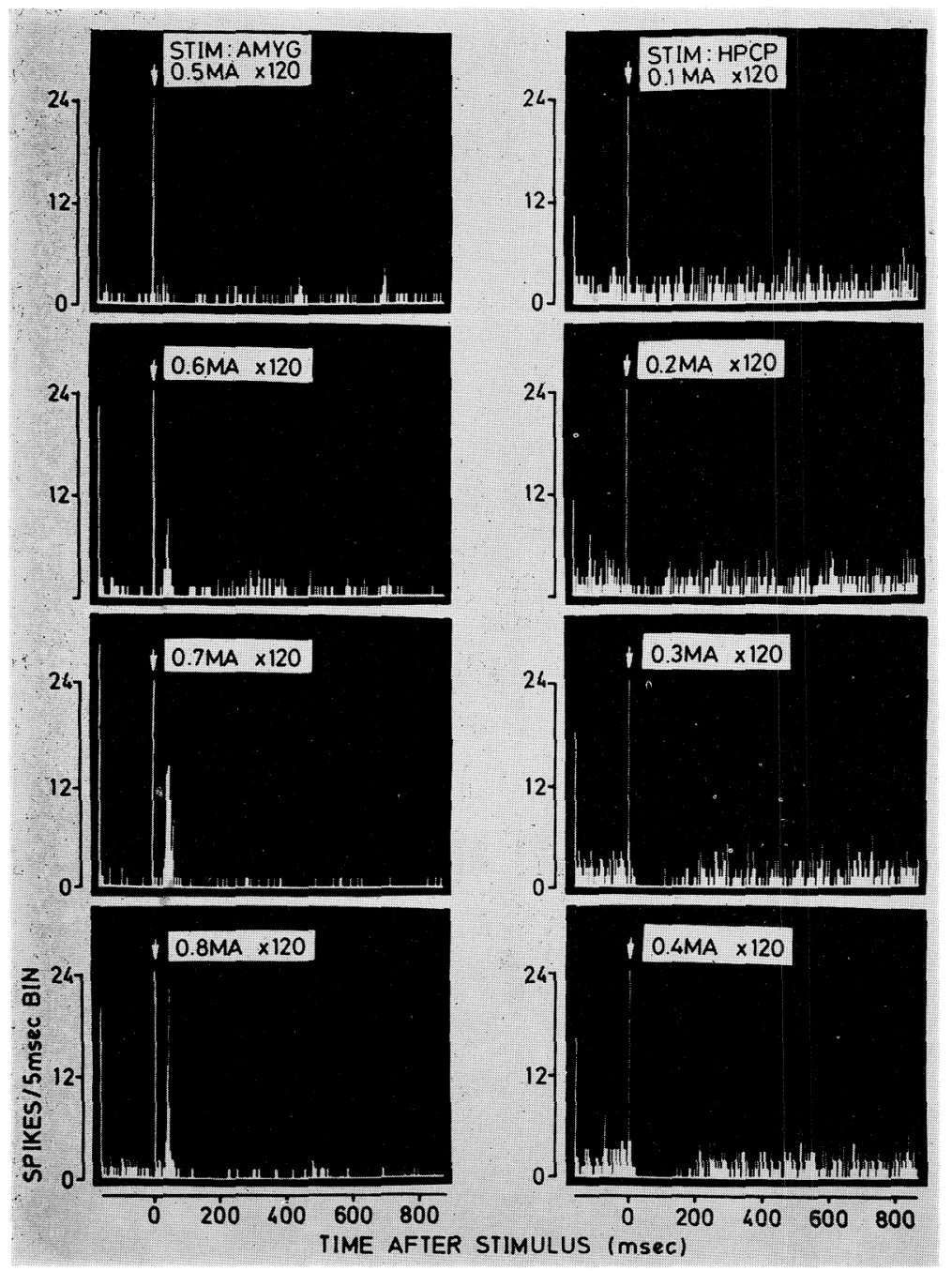

Fig. 2. Examples of poststimulus time histograms of medial preoptic neurons. responding to stimulation of the medial amygdala (left) and the dorsal hippocampus (right). Computations were repeated 120 times in each histogram. Stimulus threshold was found to be $0.6 \mathrm{~mA}$ for the amygdaloid and 0.2 $\mathrm{mA}$ for the hippocampal stimulation. The larger stimulus current elicited the larger unit response.

through a preamplifier (Nihon Koden, NZ-4) to an oscilloscope (Nihon Koden, VC-9). Single unit responses to consecutively applied 80-560 stimuli were computed on line and a post-stimulus time histogram with 5 -msec bins was made with a digital computer (San-ei Sokki, 7T-07). Unit responses were also photographed on the oscilloscope screen if necessary. The neurons recorded were classified into 3 categories (facilitatory, inhibitory and no response) according to their initial responses, since a facilitatory response was usually followed by a post-facilitatory inhibition and an inhibitory response followed by a post-inhibitory excitation. The threshold of the stimulus current was determined in some units by repeating the histogram analysis with different current intensities. Fig. 2 shows examples of the threshold determination in the preoptic neurons.

At the end of each experiment, the micropipette tip position was marked by passing cathodal current of $10 \mu \mathrm{A}$ for $10 \mathrm{~min}$. The site of stimulation was marked by passing an anodal current of $10 \mu \mathrm{A}$ for $30 \mathrm{sec}$. The animal was perfused with $10 \%$ buffered formalin and $3 \%$ potassium ferricyanide and ferrocyanide solutions, and the electrode tip positions were histologically determined in frozen sections of the brain.

The chi-square test and Fisher exact probability test were used for statistical analysis of the number of neurons showing excitations or inhibition in response. Analysis of variance followed by the Duncan multiple range test was used for comparison of the stimulus thresholds. 


\section{Results}

Hypothalamic arcuate unit responses to the medial preoptic stimulation

Spontaneously firing unit activities were recorded from 321 arcuate neurons, and all of them were tested for their transsynaptic responses to the single pulse stimulation of the medial preoptic area. In total $75(23.4 \%)$ and $47(14.6 \%)$ arcuate units respectively were facilitated and inhibited by the stimulation. The remaining $199(62.0 \%)$ units did not respond to the stimulus. The numbers and percentages of the units tested which increased, decreased or did not change their firing activities in various rats are summarized in Table 1 and Fig. 3. The numbers of arcuate neurons which responded to the preoptic stimulation were not different between young, mature and

Percentages of Hypothalamic Arcuate Neurons Responding 700 to Single Pulse Stimulation of the Medial Preoptic Area

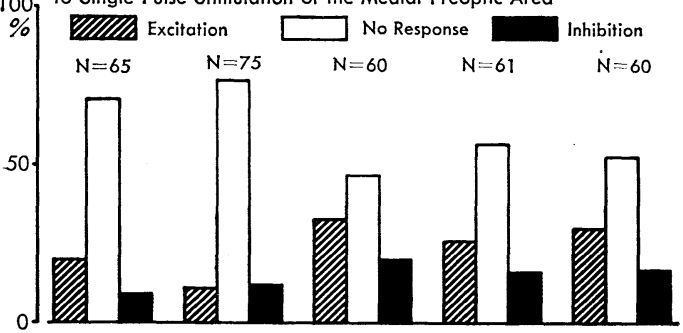

Thresholds of Preoptic Stimulation for Inducing Arcuate

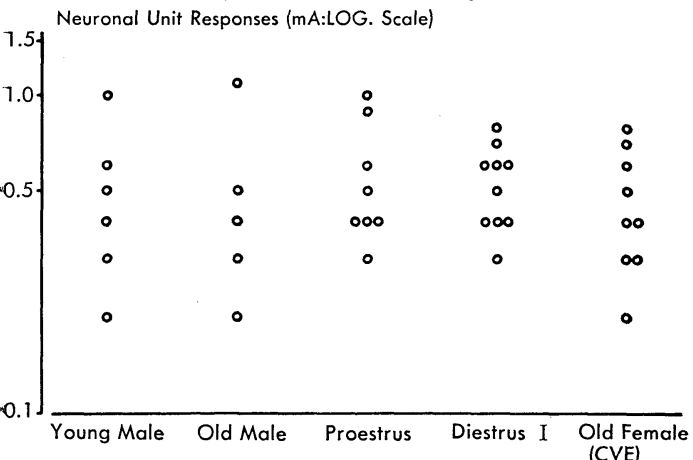

(CVE)

Fig. 3. Responses of hypothalamic arcuate neurons to the medial preoptic stimulation and the stimulus thresholds successfully determined for inducing the unit response.

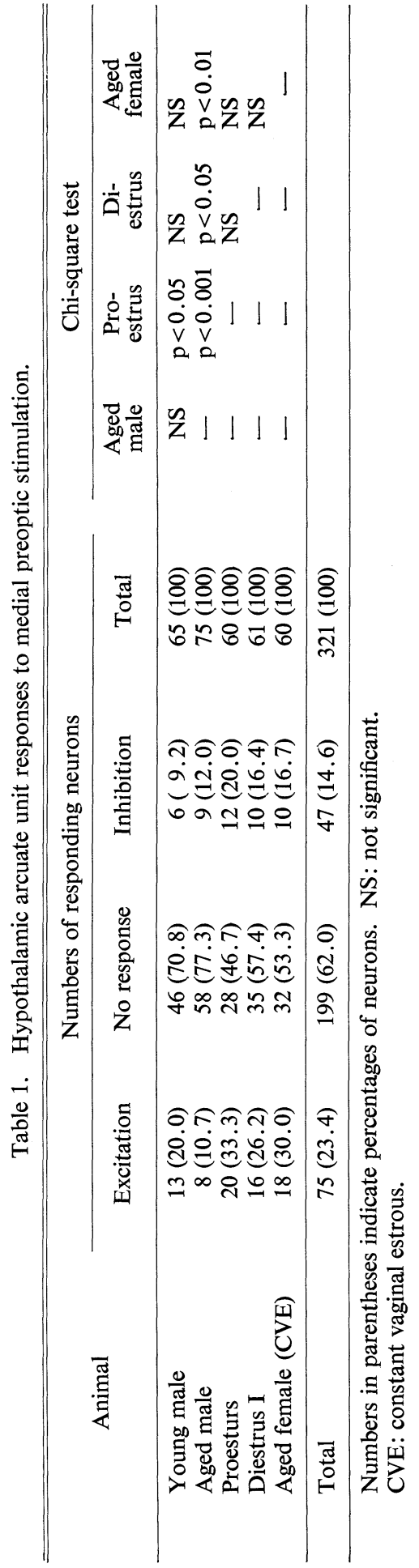


aged rats. However, there was a sex difference in the number of responsive arcuate neurons. The preoptic stimulation induced unit responses in significantly more arcuate neurons in the proestrous rat than in the young male $(\mathrm{p}<$ 0.05 ), and the stimulation induced more arcuate unit responses in the aged constant estrous rat than in the aged male $(p<0.01)$. The stimulus threshold was determined for 38 responsive neurons. There was no statistical variation in the threshold in different groups of animals (Fig. 3).

Medial preoptic unit responses to the medial amygdaloid stimulation

In various groups of rats, 536 neurons were recorded in the medial preoptic area and tested for their responses to the stimulation of the medial amygdala. They included 88 neurons in which the response to the dorsal hippocampal stimulation was also tested. The convergence of amygdaloid and hippocampal inputs to the preoptic neuron is described later.

The amygdaloid stimulation induced an

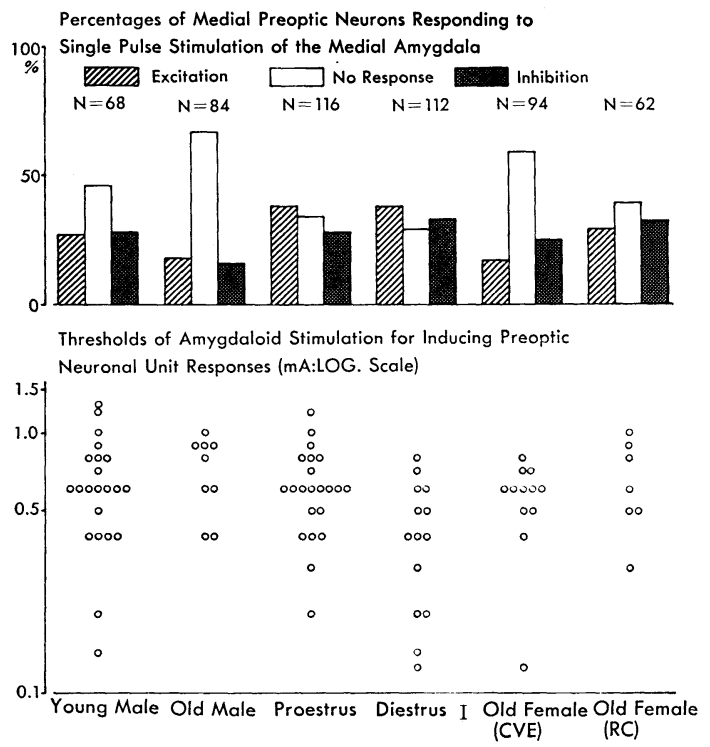

Fig. 4. Responses of medial preoptic neurons to the medial amygdaloid stimulation in various rats. Stimulus thresholds of the amygdala are also shown.

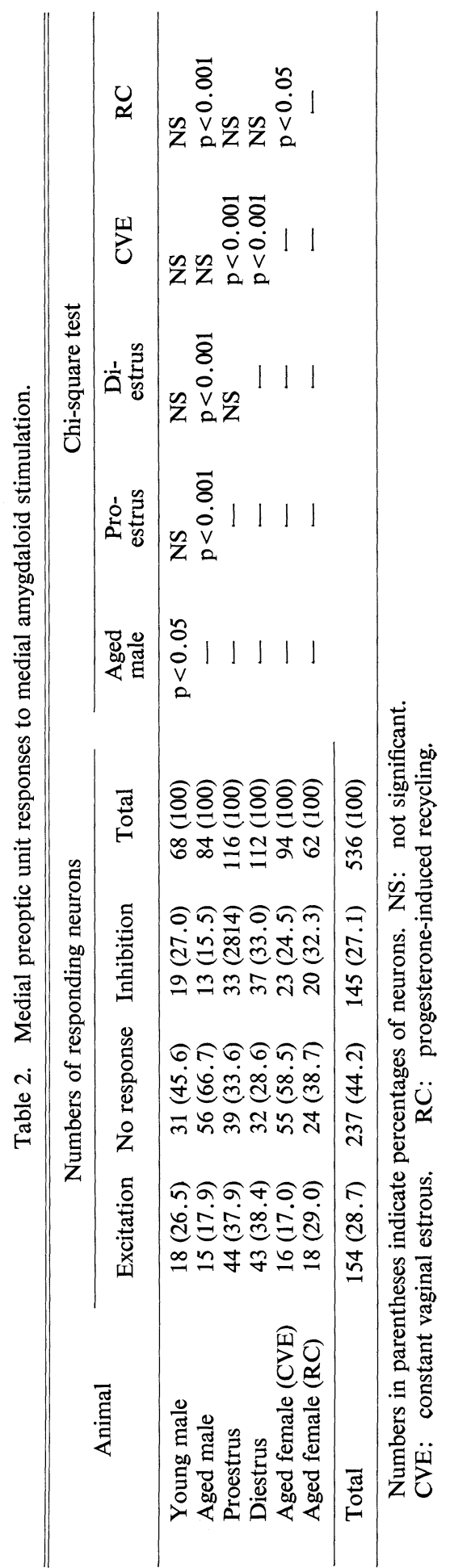


excitatory response in 154 units $(28.7 \%)$ and an inhibitory response in 145 units $(27.1 \%)$ in total. The remaining 237 units $(44.2 \%)$ were unresponsive. The proportion of preoptic neurons responding to the amygdaloid stimulation among all neurons tested was quite similar in different rats of the same group. Table 2 and Fig. 4 show the numbers and percentages of responding neurons and the threshold currents for changing the unit activity in different groups of rats. Percentages of responding neurons were not different between sexes or between proestrous and diestrous females. However, the responsive neurons were significantly decreased in aged rats of each sex as compared with young mature rats $(\mathrm{p}<0.05$ for males; $p<0.001$ for females). In old female rats whose vaginal cycles were induced by daily treatment with progesterone, the number of responsive units was significantly greater than that in constant estrous aged rats $(\mathrm{p}<$ 0.05 ), and was similar to that in young mature females. The stimulus threshold of the medial amygdala for inducing preoptic unit response was quite similar in different groups (Fig. 4).

Medial preoptic unit responses to the hippocampal stimulation

Five hundred and six spontaneously firing neurons recorded in the medial preoptic area were tested for their responses to electrical stimulation of the dorsal hippocampus. Among them $49(9.7 \%)$ and $142(28.1 \%)$ units showed an excitatory and an inhibitory response to the stimulation, respectively. Table 3 and Fig. 5 show the numbers and percentages of units responding to the hippocampal stimulation and the thresholds of the stimulation for inducing preoptic unit responses in different animal groups

Proestrous rats had significantly more preoptic neurons which responded to the hippocampal stimulation than young males $(\mathrm{p}<$ $0.001)$ or diestrous rats $(\mathrm{p}<0.001)$. Excitatory responses were more frequently recorded than inhibitory responses in the male, while inhibitory responses were more frequent in diestrous

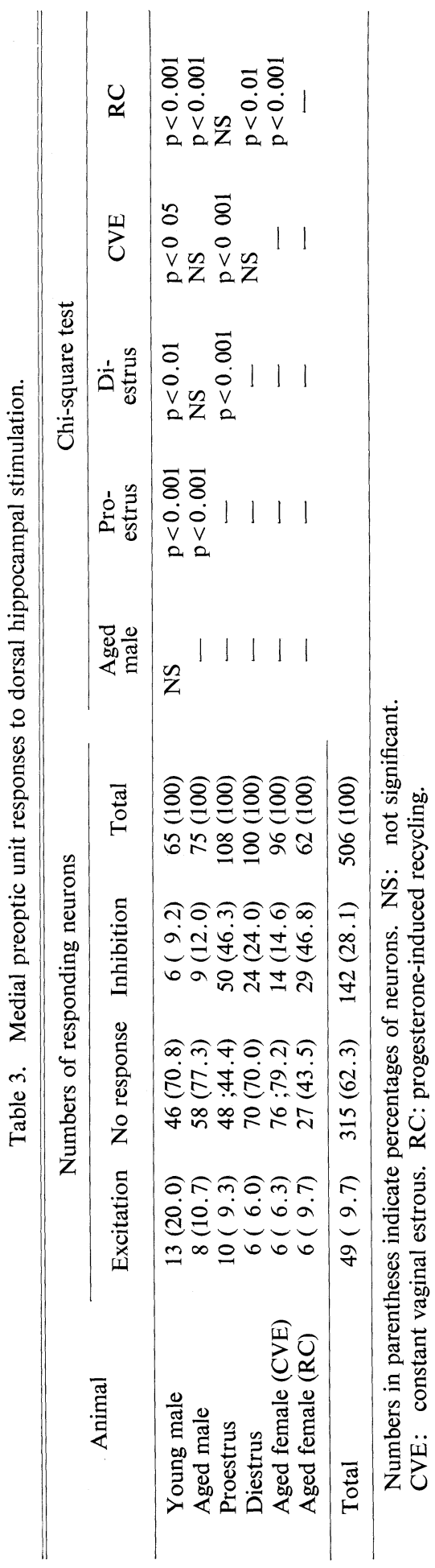






Fig. 5. Responses of medial preoptic neurons to the dorsal hippocampal stimulation in different endocrine conditions. Stimulus thresholds of the hippocampus are also shown.

rats, though the total number of responding units was similar in males and diestrous females. The preoptic unit response to the hippocampal stimulation was thus statistically different among these 3 groups of young rats depending on the sex and the state of the estrous cycle (Table 3).

In aged constant estrous rats, only onefifth or the preoptic neurons responded to stimulation of the hippocampus; the proportion of the responding neurons was significantly smaller than that in young proestrous rast $(\mathrm{p}<0.001)$. On the day of proestrous in re- cycling aged rats whose estrous cycles were induced by daily injection of progesterone, the hippocampal stimulation induced changes in firing activity in more than a half of the preoptic neurons tested. Thus, the daily progesterone treatment resulted in almost complete restoration of the preoptic unit responsiveness in old constant estrous rats $(\mathrm{p}<0.001)$, as well as the restoration of the estrous cycle (Fig. 1).

The stimulus thresholds of the dorsal hippocampus were similar in different animal groups in spite of the difference in the number of responding neurons (Fig. 5).

\section{Convergence of amygdaloid and hippocampal afferents to preoptic units}

In rats under various endocrine conditions, 143 neurons in the medial preoptic area were tested for their responses to stimulation of the medial amygdala and the dorsal hippocampus. Fifty-five of them were recorded in ovariectomized rats with or without estrogen treatment $(20 \mu \mathrm{g}$, injected s.c. 2 days before use). The results summarized in Table 4 indicate that all possible response combinations were recorded. Various types of response combinations are shown in Fig. 6. Four-fifths of the neurons tested changed their firing activities in response to stimulation of either area, including one-fourths of all responding to both. In the remaining one-fifth, stimulation of neither area was effective.

\section{Discussion}

The post-stimulus histogram analysis re-

Table 4. Medial preoptic unit responses to amygdaloid and hippocampal stimulation.

\begin{tabular}{llrrrr}
\hline \hline & & \multicolumn{4}{c}{ Responses to amygdaloid stimulation } \\
\cline { 3 - 5 } & & Excitation & No response & Inhibition & Total \\
\hline Responses to & Excitation & $10(7.0)$ & $9(6.3)$ & $8(5.6)$ & $27(18.9)$ \\
Hippocampal & No response & $32(22.4)$ & $28(19.6)$ & $28(10.6)$ & $88(61.5)$ \\
stimulation & Inhibition & $8(5.6)$ & $9(6.3)$ & $11(7.7)$ & $28(19.6)$ \\
& Total & $50(35.0)$ & $46(32.2)$ & $47(32.9)$ & $143(100.0)$ \\
\hline
\end{tabular}

Numbers in parentheses indicate percentages of neurons. 


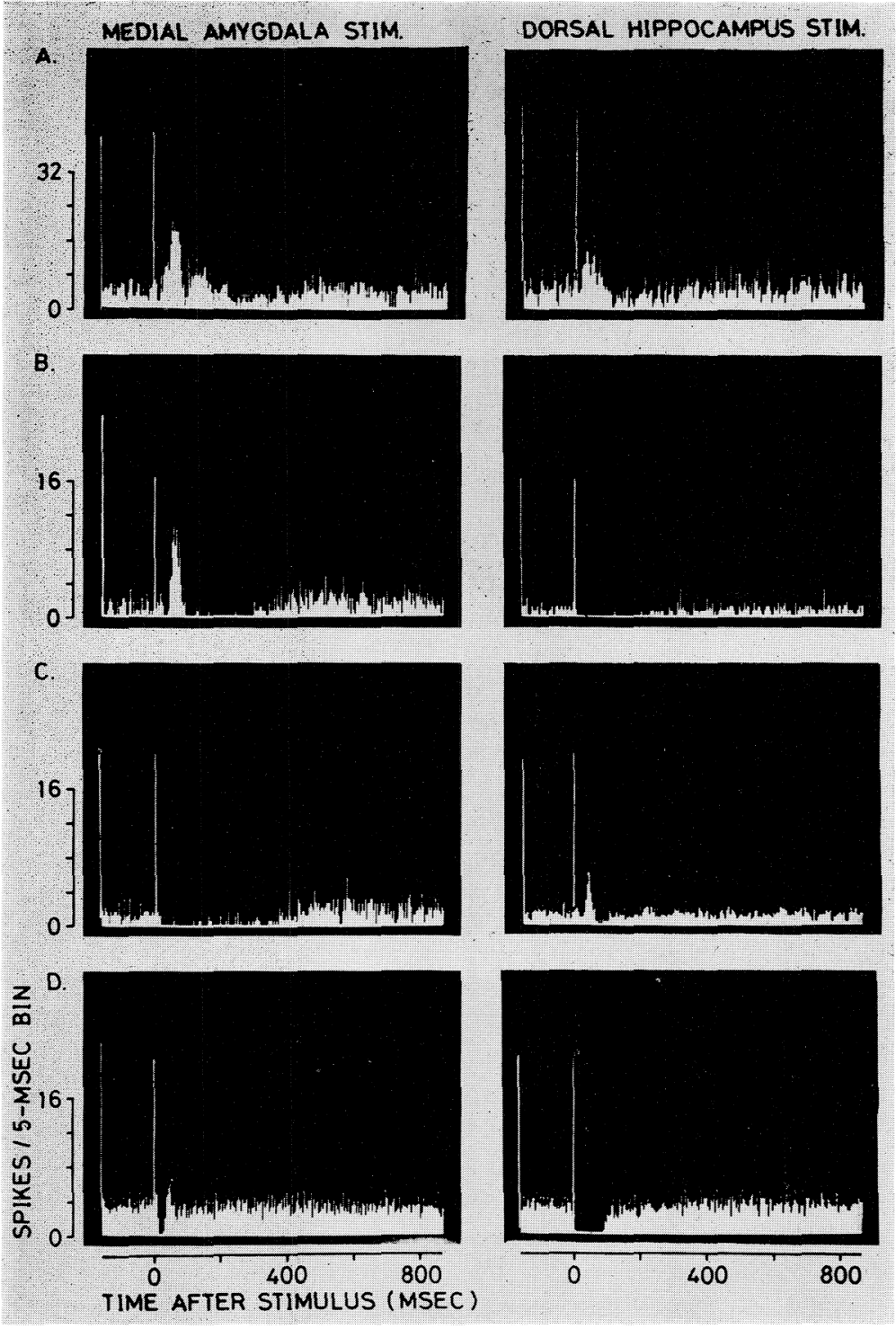

Fig. 6. Various types of response combinations of media! preoptic neurons to electrical stimulation of the medial amygdala and the dorsal hippocampus. All possible response combinations were recorded, suggesting a complexity of convergences of the limbic fibers on preoptic neurons.

vealed that the medial preoptic stimulation modified spontaneous spike activities in $38 \%$ of neurons recorded in the hypothalamic arcuate nucleus. The female rat was found to have a larger number of arcuate neurons responsive to the preoptic stimulation than the male. That a change in neuronal excitability in the site of preoptic stimulation resulted in the difference in the arcuate unit response is not likely, since the threshold of the stimulation for inducing the unit response was quite similar in males and females. Alternatively, the synaptic function for transmitting preoptic impulses to arcuate neurons seems to be sexually different. Matsumoto and Arai (1980) reported that the arcuate neurons in the female rat have more axo-dendritic synapses than those in the male, while axo-somatic synapses 
are more frequently found in the male than in the female. The formation of these morphological dimorphisms is dependent on the exposure of the brain to gonadal steroid hormones (Matsumoto and Arai, 1980). There may be some functional relationship between these morphological difference and our unit response data.

There are direct axonal projections from the preoptic-anterior hypothalamic area to the medial basal hypothalamus, including the arcuate and the ventromedial nuclei (Dyer, 1973; Dyer, et al., 1976; Kawakami and Sakuma, 1974). The preoptic anterior hypothalamic neurons projecting their axons to the medial basal hypothalamus receive sexually differentiated inputs from the amygdala. According to Dyer et al. (1976), significantly more of these neurons receive synaptic connections from the amygdala in the male than in the female. These neurons send their axons to the medial basal hypothalamic neurons and would regulate the activity of the latter neurons. The sexual difference in the arcuate unit response to the preoptic stimulation may suggest a sexually differentiated synaptic output of preoptico-tuberal fibers in addition to their sexually dimorphic input from the amygdala.

About four-fifths of the medial preoptic neurons responded to stimulation of one or both of the medial amygdala and the dorsal hippocampus. Convergence of the amygdaloid and hippocampal fibers was observed in onefourth of the medial preoptic neurons recorded. The preoptic cells also receive afferent impulses from the medial basal hypothalamus (Dyer, 1973; Dyer, et al., 1976; Fenske, et al., 1975), median eminence (Yagi and Sawaki, 1977), medial or mediocortical amygdala (Dyer, et al., 1976; Fenske, et al., 1975; Kawakami and Kubo, 1971), lateral amygdala, medial and lateral septum, ventral part of the midbrain reticular formation, dorsomedial thalamic nucleus and babenular nuclei (Kawakami, et al., 1979), dorsal hippocampus (Kawakami and Kubo, 1971; Kawakami, et al., 1979), medial forebrain bundle (Perkins and
Whitehead, 1978), midbrain raphe (Fenske, et al., 1975), and dorsal tegmentum (Carrer and Sawyer, 1976). Although the functional implications of these convergent inputs to the preoptic area are still speculative, an integral role of the neurons in this area is possible.

In the present experiment, we observed that $69 \%$ of the medial preoptic cells are responsive to stimulation of the medial amygdala, which is $15 \%$ less than that reported by Fenske et al. (1975) and 15\% more than the result obtained by Dyer et al. (1976). Facilitatory responses were more frequently observed than inhibitory responses. Dyer et al. (1976) reported that significantly more of the preopticanterior hypothalamic cells projecting directly to the medial basal hypothalamus receive synaptic connections arising from the amygdala in males than in females. However, such a sexual difference in the preoptic unit response to the amygdaloid stimulation was not observed in the present experiment. Although the reason for this discrepancy between their results and ours is not certain, it may be related to the difference in the neurons sampled in each experiment. No attempt was made in our study to identify the preoptic neurons projecting their axons to the medial basal hypothalamus, while Dyer et al. (1976) recorded unit responses from antidromically identified units. Morphologically, the number of non-amygdaloid synapses on dendritic spines of the preoptic neuron in female rats is significantly larger than that in males (Raisman and Field, 1973). According to Dyer et al. (1976) $27 \%$ of spontaneously firing preoptic-anterior hypothalamic units send their axons to the medial basal hypothalamus. Therefore, the amygdaloid afferents terminating on the majority of preoptic neurons which do not project to the medial basal hypothalamus seem to have no sexual differentiation in the axonal input from the amygdala.

On the other hand, responses of preoptic units to dorsal hippocampal stimulation were more frequently recorded in proestrous rats than in diestrous or young male rats. Excita- 
tory responses were more frequently observed than inhibitory responses in male rats while inhibitory responses were more frequent in proestrous or diestrous females. Although the basic mechanism forming these functional changes is not certain, the higher incidence of preoptic unit responses in proestrous females may be related to the ultrastructural dimorphism found by Raisman and Field (1973). However, the number of preoptic units responding to the hippocampal stimulation in female rats was reduced significantly on the day of diestrous when compared to the day of proestrous. It is difficult to postulate that the morphology, namely the number of synapses, in the preoptic area may change during the estrous cycle. Alternatively, it must be taken into account that the amounts of catecholamine transmitters in the hypothalamus, including the preoptic area, fluctuate during the estrous cycle and become significantly higher on the day of proestrous (Kordon, et al., 1976). The threshold current of the hippocampal stimulation needed for inducing preoptic unit responses was quite similar in proestrous and diestrous rats. Therefore, the reduction in the preoptic unit responses in diestrous rats may be due to a relative loss of hypothalamic transmitter activities rather than a change in excitability of the hippocampal neurons.

Significantly less neurons in the medial preoptic area were responsive to amygdaloid stimulation in aged rats than in young mature animals in each sex. However, the stimulus threshold of the amygdala requisite for the induction of preoptic neuronal responses was almost the same. Similarly, preoptic unit responses to the hippocampal stimulation were significantly reduced in aged constant estrous females without any obvious change in the stimulus threshold. The hippocampus-induced response, however, was not clearly reduced in aged male rats when compared to young males, presumably due the fact that the percentage of preoptic neurons responding to the hippocampal stimulation was already as low in young males as in aged rats. The hypothalamic catecholamine contents are significantly lower in aged rats (Simpkins, et al., 1977) and mice (Finch, 1973) than in young animals of the same species. These results may imply that the senile loss of impulse-transmitting activity of the amygdalo-preoptic and hippocampo-preoptic fibers is brought about by the senile change in chemical transmitter levels within these structures. However, a change in synaptic morphology or in the postsynaptic responsiveness to the transmitter cannot be ruled out.

Stimulation of the preoptic area causes a rise in serum gonadotropin concentrations and ovulation equally in pentobarbital-blocked proestrous rats (Kawakami, et al., 1973b) and in aged constant estrous rats (Clemens, et al., 1969). Since the latter animals do not ovulate spontaneously, the afferent inputs which regulate the activation of preoptic neurons may be functionally modified in the aged rats in association with the loss of the normal occurrence of ovulation. Daily treatment with progesterone induced vaginal cycles in aged constant estrous rats. Progesteroneinduced reinitiation of estrous cycles has been reported earlier (Everett, 1940; Huang, et al., 1976). Huang et al. (1976) suggested, among other possibilities, that progesterone may increase hypothalamic catecholamine activity in aged constant estrous rats to stimulate LHRH release and ovulation. In the present study it was found that the responsiveness of preoptic neurons to the amygdaloid and hippocampal stimulation, which had been very low in aged constant estrous rats, was completely restored following daily progesterone treatment which induced estrous cycles. This result suggest a possible relationship between the function of the limbic-preoptic synaptic connections and the ability of the young but not the aged female rat to induce cyclic gonadotropin release and ovulation.

\section{References}

Albe-Fessard, D., F. Stutinsky et S. Libouban (1966). Atlas stereotaxique du Diencephale du Rat Blanc. 
C.N.R.S., Paris.

Carrer, H. F. and C. H. Sawyer (1976). Changes in multiunit spike activity in the rat preoptic area induced by stimulating the midbrain. Exp. Neurol. 52, 525-534.

Clemens, J. A., Y. Amenomori, T. Jenkins and J. Meites (1969). Effects of hypothalamic stimulation, hormones, and drugs on ovarian function in old female rats. Proc. Soc. Exp. Biol. Med. 132, 561563.

Dyer, R. G. (1973). An electrophysiological dissection of the hypothalamic regions which regulate the pre-ovulatory secretion of luteinizing hormone in the rat. J. Physiol. (Lond.) 234, 421-442.

Dyer, R. G., N. K. MacLeod and F. Ellendorff (1976). Electrophysiological evidence for sexual dimorphism and synaptic convergence in the preoptic and anterior hypothalamic area of the rat. Proc. Roy. Soc. Lond. B. 193, 421-440.

Everett, J. W. (1940). The restoration of ovulatory cycles and corpus luteum formation in persistentestrous rats by progesterone. Endocrinology 27, 681-686.

Everett, J. W. and H. M. Radford (1961). Irritative deposits from stainless steel electrodes in the preoptic rat brain causing release of pituitary gonadotropin. Proc. Soc. Exp. Biol. Med. 108, 604-609.

Fenske, M., F. Ellendorff and W. Wuttke (1975). Response of medial preoptic neurons to electrical stimulation of the mediobasal hypothalamus, amygdala and mesencephalon in normal, serotonin or catecholamine deprived female rats. Exp. Brain Res. 22, 495-507.

Finch, C. E. (1973). Catecholamine metabolism in the brains of aging male mice. Brain Res. 52, 261276.

Huang, H. H., S. Marshall and J. Meites (1976). Induction of estrous cycles in old non-cycling rats by progesterone, ACTH, ether stress or L-dopa. Neuroendocrinology 20, 21-34.

Kawakami, M. and K. Kubo (1971). Neuro-correlate of limbic-hypothalamo-pituitary gonadal axis in the rat: changes in limbic-hypothalamic unit activity induced by vaginal and electrical stimulation. Nenroendocrinology 7, 65-89.

Kawakami, M. and Y. Sakuma (1974). Responses of hypothalamic neurons to the microiontophoresis of LH-RH, LH and FSH under various levels of circulating ovarian hormones. Neuroendocrinology
15, 290-307.

Kawakami, M., F. Kimura and T. Higuchi (1973a) Effects of electrical stimulation of the brain on gonadotropin secretion in male rats. Endocrinol. Japon. 20, 447-454.

Kawakami, M., E. Terasawa, F. Kimura and K. Wakabayashi (1973b). Modulating effect of limbic structures on gonadotropin release. Neuroendocrinology 12, 1-16.

Kawakami, M., T. Akema and S. Ando (1979). Electrophysiological studies on the neural networks. among estrogen and progesterone effective brain areas on lordosis behavior of the rat. Brain Res. 169, 287-301.

Kordon, C., J. Epelbaum, A. Engerbert and J. McKelvy (1976). Neurotransmitter interactions with neuroendocrine tissue. In F. Naftolin, K. J. Ryan and J. Davies (eds.) Subcellular Mechanisms in Reproductive Neuroendocrinology. Elsevier, Amsterdom, pp. 167-184.

Matsumoto, A. and Y. Arai (1980). Sexual dimorphism in 'wiring pattern' in the hypothalamic arcuate nucleus and its modification by neonatal hormonal environment. Brain Res. 190, 238-242.

Peng, M. T. and H. H. Huang (1972). Aging of hypothalamic-pituitary-ovarian function in the rat. Fertil. Steril. 23, 535-542.

Perkins, M. N. and S. A. Whitehead (1978). Responses and pharmacological properties of preoptic/ anterior hypothalamic neurones following medial forebrain bundle stimulation. I. Physiol. (Lond.) 279, 347-360.

Quinn, D. L. (1966). Luteinizing hormone release following preoptic stimulation in the male rat. Nature (Lond.) 209, 891-892.

Raisman, G. and P. M. Field (1973). Sexual dimorphism in the neuropil of the preoptic area of the rat and its dependence on neonatal androgen. Brain Res. 54, 1-29.

Simpkins, J. W., G. P. Mueller, H. H. Huang and J. Meites (1977). Evidence for depressed catecholamine and enhanced serotonin metabolism in aging. male rats: possible relation to gonadotropin secretion. Endocrinology 100, 1672-1678.

Yagi, K. and Y. Sawaki (1977). Medial preoptic nucleus neurons: inhibition and facilitation of spontaneous activity following stimulation of the median eminence in female rats. Brain Res. 120, 342-346. 